\title{
SIMULAÇÃO DO PERFIL DE VELOCIDADES E PRESSÃO EM UM MEDIDOR DE VAZÃO TIPO VENTURI POR MEIO DE UM SOTWARE DE SIMULAÇÃO CFD
}

\author{
A. B. de ALMEIDA ${ }^{1}$, C. I. YAMAMOTO ${ }^{2}$, L. F. L. LUZ JR. ${ }^{2}$ \\ ${ }^{1}$ Universidade Federal do Paraná, Escola Piloto de Engenharia Química da UFPR \\ ${ }^{1}$ Universidade Federal do Paraná, Departamento de Engenharia Química \\ E-mail para contato: amandaborges.almeida@gmail.com
}

\begin{abstract}
RESUMO - O presente trabalho tem como objetivo simular os perfis de velocidade e pressão do medidor de vazão tipo Venturi do Laboratório de Engenharia Química da Universidade Federal do Paraná empregando um modelo computacional desenvolvido com a ferramenta CFD, utilizando o programa ANSYS CFX 15.0. O intuito principal é contribuir para as aulas práticas realizadas no curso facilitando o entendimento dos alunos e facilitando o processo de calibração do instrumento, pois, uma vez validada, a simulação representa muito bem a realidade. As vazões experimentais foram obtidas pela diferença entre as pressões em diferentes regiões do tubo Venturi utilizando um manômetro de mercúrio. Os resultados de velocidade, calculados a partir dos dados experimentais, são comparados com os obtidos a partir da simulação, verificandose baixos erros de medição, menores de $2 \%$. Determinou-se também o melhor tipo de malha para a geometria de um Venturi. Para isso, realizou-se a simulação utilizando uma malha estruturada e uma não estruturada e os resultados de ambas as simulações são comparados com os experimentais. A malha não estruturada apresentou resultados mais precisos, no entanto os tempos de simulação foram bem maiores.
\end{abstract}

\section{INTRODUÇÃO}

O medidor Venturi se constitui em um dos métodos mais utilizados para a medição de vazão em tubos, pois têm a vantagem de apresentar baixas perdas de carga porque não ocorre a separação de uma camada de fluido turbulenta, como ocorre na placa de orifício. Outra característica desse medidor é que são auto limpantes, podendo medir a vazão de fluidos com grande quantidade de sedimentos (Fox, 2014).

Assumindo que o escoamento no Venturi é unidimensional e não viscoso, pode-se aplicar a Equação de Bernoulli nas seções em que se localizam as tomadas de pressão. Essa equação prevê um aumento da velocidade do fluido a medida que a seção diminui. Simultaneamente ocorre uma diminuição na pressão ou diminuição na energia potencial do fluido. Combinando a equação de Bernoulli, com a equação da continuidade e conhecendo-se apenas a queda de pressão no dispositivo, a vazão mássica pode ser avaliada para escoamento idealizado incompressível por meio da Equação (1), que expressa a vazão mássica $m \mathrm{em} \mathrm{kg} / \mathrm{s}$ 
em função da carga diferencial de líquido $\Delta h(\mathrm{~m})$ correspondente a diferença de pressão do mesmo em um ponto situado antes e no estreitamento (Perry, 1999).

$$
m=A_{2} \cdot \rho \cdot \sqrt{\frac{2 \cdot g \cdot \Delta h}{1-\beta^{4}}}
$$

onde $S_{2}$ é a área transversal do estreitamento e $\beta$ o quociente de diâmetros da condução e do estreitamento.

No entanto, é comum na literatura a incorporação de um fator de correção, " $\mathrm{C}_{\mathrm{D}}$ " (Coeficiente de Descarga), para considerar a não idealidade do sistema, ou seja, as perdas por atrito. Deste modo, obtém-se a equação básica de projeto aplicada quando se tem fluidos incompressíveis:

$$
m=A_{2} \cdot \rho \cdot C_{D} \sqrt{\frac{2 \cdot g \cdot \Delta h}{1-\beta^{4}}}
$$

Esse coeficiente depende tanto do número de Reynolds como do parâmetro $\beta$, e encontra-se tabulado ou representado graficamente para diferentes tipos de medidores de vazão. Nos Venturis, por não haver contração da vena contracta depois da sua garganta os coeficientes de descarga são mais elevados e podem ser considerados independentes de $\beta$ (Bird, 2004).

ANSYS CFX é um software de Fluidodinâmica Computacional, que combina um solucionador avançado com capacidades poderosas de pré e de pós-processamento. Ele inclui um solver confiável e robusto, a integração completa de definição do problema, análise e apresentação de resultados e um processo de configuração intuitiva e interativa. (CFX User's Manual).

Neste trabalho, as simulações numéricas foram realizadas através do código comercial CFX, com respaldo da licença de uso junto ao fornecedor ANSYS, com o intuito de determinar as particularidades do escoamento no interior que um medidor de vazão do tipo Venturi. Deste modo, será possível contribuir para as aulas práticas realizadas no curso e os estudantes terão a oportunidade de observar o que ocorre no interior do instrumento como, por exemplo, a conservação de energia. Além disso, também se pretende diminuir os gastos e tempo com experimentos que tem como objetivo a calibração do Venturi, já que isso será possível fazer empregando a simulação.

Apesar de ser um exemplo que pode ser considerado simples, não se encontrou muitos trabalhos na literatura que comparam resultados de CFD e dados experimentais em medidores tipo Venturi. Dias et al. (2009) validou, por meio de ensaio experimental, o modelo computacional criado com o CFD de um tubo Venturi do Laboratório Hidromecânico para Pequenas Centrais Hidrelétricas da Universidade Federal de Itajubá. Esse autor empregou malhas não estruturadas e variou o refinamento das mesmas. Além disso, realizou simulações alterando o modelo de turbulência. Esse autor comparou vazões e os erros foram menores de $2 \%$. Sendo que a malha mais refinada e o modelo de turbulência $\mathrm{k}-\varepsilon$ foram os que apresentarão melhor precisão dos resultados (erro de apenas 1,6\%).

\section{MATERIAIS E MÉTODOS}


A instalação experimental é simples e consta de um Venturi e de um manômetro de mercúrio conectado no inicio do cone convergente e na garganta do instrumento. A medida de pressão é possível através da utilização de anéis piezométricos. Por meio de uma bomba centrífuga, bombeia-se água pela tubulação e, variado a potência da bomba, é possível variar a vazão que circula pelo instrumento. Na Figura 1 é possível observar o módulo experimental:

Figura 1 - Instalação experimental

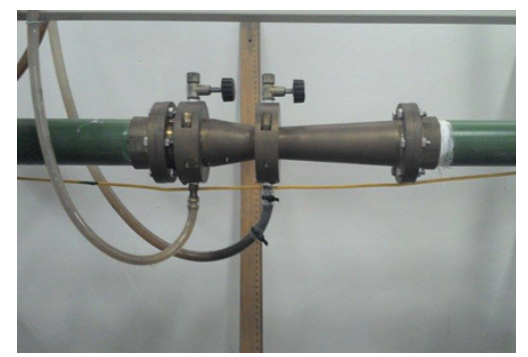

A razão entre os diâmetros da obstrução e da canalização $(\beta)$ é 0,6 . Sabendo que o diâmetro da canalização é $40,5 \mathrm{~mm}$, sabe-se que o diâmetro da obstrução, $D_{2}$, é $24,3 \mathrm{~mm}$. $\mathrm{O}$ comprimento da garganta, medido no laboratório, é de $25 \mathrm{~mm}$. Além disso, com os valores da angulação do cone convergente de entrada de $21^{\circ}$ e divergente de saída de $14^{\circ}$, é possível reproduzir com exatidão a geometria do Venturi. O coeficiente de descarga deste Venturi, necessário na simulação para o cálculo das vazões (Equação 2), já foi determinado previamente é igual a 0,9706 .

A geometria foi feita no programa ICEM CFD 15.0, software do pacote ANSYS, respeitando as dimensões do tubo Venturi cujo perfil de velocidades e pressões se deseja conhecer e, com o mesmo programa, criou-se uma malha estruturada (Figura 2) utilizando o recurso de "blocagem". Além disso, com o intuito de verificar o melhor tipo de malha para essa simulação, criou-se uma malha não estruturada utilizando os recursos do mesmo software. Os detalhes dessa malha podem ser observados na Figura 3.

A malha estruturada da Figura 2 possui 252720 nós e 24316 elementos e a não estruturada da Figura 3 possui 69790 nós e 386463 elementos.

Figura 2 - Malha estruturada (Malha 1)
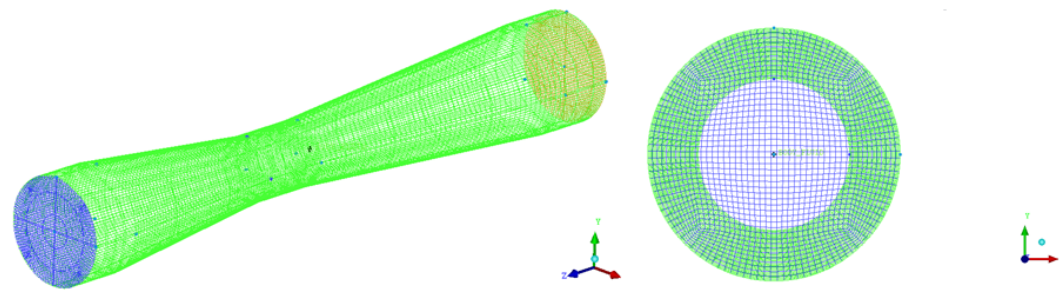
Figura 3 - Malha não estruturada
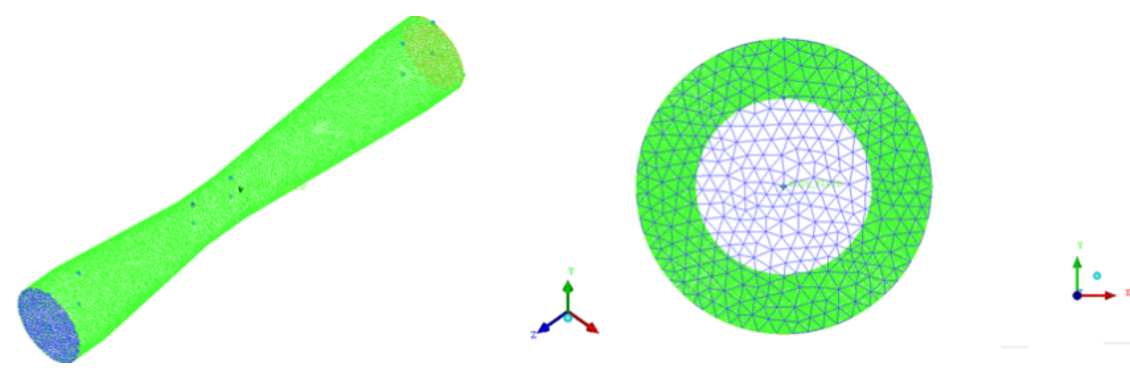

\section{RESULTADOS E DISCUSSÃO}

Na Tabela 1, é possível observar os resultados de vazão e velocidades na garganta determinadas experimentalmente. Esses dados representam as médias dos pontos medidos experimentalmente, tomados em quadruplicata.

Tabela 1 - Resultados experimentais

\begin{tabular}{cccccc}
\hline Ponto & $\begin{array}{c}\Delta \mathrm{h} \\
(\mathrm{m})\end{array}$ & $\begin{array}{c}\Delta \mathrm{P} \\
\left(\mathrm{N} / \mathrm{m}^{2}\right)\end{array}$ & $\begin{array}{c}\mathrm{m} \\
(\mathrm{kg} / \mathrm{s})\end{array}$ & $\begin{array}{c}\mathrm{v}_{1} \\
(\mathrm{~m} / \mathrm{s})\end{array}$ & $\begin{array}{c}\mathrm{v}_{2} \text { Experimental } \\
(\mathrm{m} / \mathrm{s})\end{array}$ \\
\hline 1 & $0,0120 \pm 0,0006$ & 117,3668 & 0,2232 & 0,1738 & 0,471 \\
2 & $0,0280 \pm 0,0006$ & 273,8560 & 0,3409 & 0,2654 & 0,719 \\
3 & $0,0550 \pm 0,0009$ & 537,9314 & 0,4778 & 0,3720 & 1,008 \\
4 & $0,0930 \pm 0,0022$ & 909,5930 & 0,6213 & 0,4837 & 1,311 \\
5 & $0,1470 \pm 0,0011$ & 1437,7438 & 0,7811 & 0,6082 & 1,648 \\
\hline
\end{tabular}

Para a simulação definiu-se como fluido a água. As propriedades do fluido já estão prédefinidas no banco de dados do programa. Como condições de contorno definiram-se as vazões mássicas que foram determinadas experimentalmente. Sendo assim, definiram-se as fronteiras de entrada, saída e de parede. Considerou-se o sistema isotérmico com uma temperatura de $25^{\circ} \mathrm{C}$ e como modelo de turbulência definiu-se o k-epsilon (padrão), que é um modelo de turbulência semi-empírico com turbulência de baixa intensidade (5\%). Na Tabela 2 é possível observar os resultados da simulação com os respectivos tempos e erros percentuais.

Tabela 2 - Resultados da simulação

\begin{tabular}{ccccrrr}
\hline Ponto & $\begin{array}{c}\mathrm{v}_{2} \text { CFX } \\
\text { Malha 1 } \\
(\mathrm{m} / \mathrm{s})\end{array}$ & $\begin{array}{c}\text { Tempo } \\
(\mathrm{s})\end{array}$ & $\begin{array}{c}\text { Erro 1 } \\
(\%)\end{array}$ & $\begin{array}{c}\mathrm{v}_{2} \text { CFX } \\
\text { Malha } 2 \\
(\mathrm{~m} / \mathrm{s})\end{array}$ & $\begin{array}{c}\text { Tempo } \\
(\mathrm{s})\end{array}$ & $\begin{array}{c}\text { Erro 2 } \\
(\%)\end{array}$ \\
\hline 1 & 0,497 & 403 & 1,371 & 0,473 & 437 & 0,859 \\
2 & 0,744 & 257 & 1,595 & 0,740 & 544 & 0,393 \\
3 & 1,042 & 244 & 1,664 & 1,038 & 436 & 0,716 \\
4 & 1,360 & 229 & 1,636 & 1,357 & 361 & 1,012 \\
5 & 1,739 & 228 & 1,575 & 1,737 & 338 & 1,295 \\
\hline
\end{tabular}


Analisando a Tabela 2 é possível observar que os resultados obtidos com a simulação são bem parecidos com os experimentais, pois se obteve um erro máximo de 1,66\%, comprovando a eficácia dos resultados obtidos por CFD. Observa-se também, que a malha não estruturada fornece resultados mais precisos, o que já se esperava, pois esse tipo de malha se adapta mais facilmente à geometria, no entanto os tempos de simulação são bem maiores (praticamente o dobro). Portanto, analisando a Tabela 2, torna-se evidente a vantagem de uma malha estruturada: os tempos de simulação são bem menores.

Observa-se também que quanto maior a vazão, maior o erro para o caso da malha não estruturada. Para verificar se esse maior erro é devido à intensidade de turbulência repetiu-se a simulação para os pontos 4 e 5 empregando uma intensidade de turbulência elevada $(10 \%)$, porém os erros continuaram elevados: 1,56 e 1,43\% respectivamente. Portanto, os erros mais elevados são devido aos maiores desvios padrão dos últimos pontos experimentais.

Na seguinte figura é possível observar o perfil de velocidades para o ponto 5 obtido com a malha estruturada:

Figura 4 - Perfil de velocidades para a vazão do ponto 5

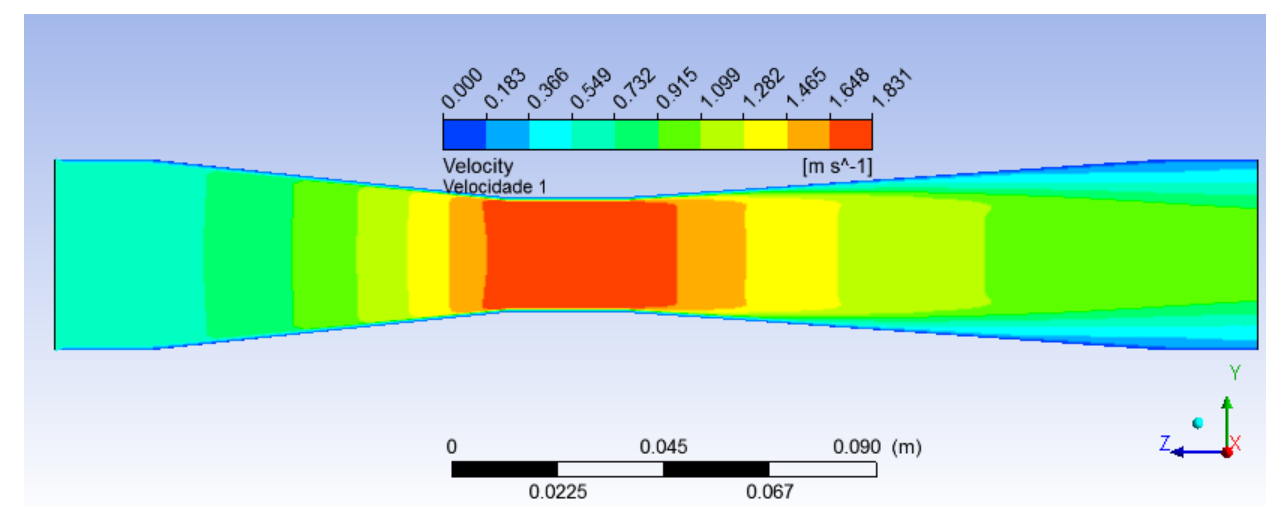

Na Figura 4 é possível observar como a velocidade é máxima na garganta, e que a medida que a tubulação volta ao diâmetro normal da condução, a velocidade se aproxima da inicial. Essa velocidade na saída do Venturi não é igual à velocidade na entrada devido à perda de carga. Na Figura 5, é possível observar o perfil de pressões onde a perda de carga se torna mais evidente.

Comparando as Figuras 4 e 5 é possível observar claramente a conversão de energia imposta pela equação de Bernoulli. Na seção convergente, antes da garganta, ocorre conversão de energia de pressão em energia cinética, pois a restrição de área provoca a aceleração do fluido a custa da sua energia de pressão. Já na seção divergente, energia cinética é convertida em energia de pressão, ocorrendo a desaceleração do fluido devido ao aumento da seção transversal da tubulação. 
Figura 5 - Perfil de pressões para a vazão do ponto 5

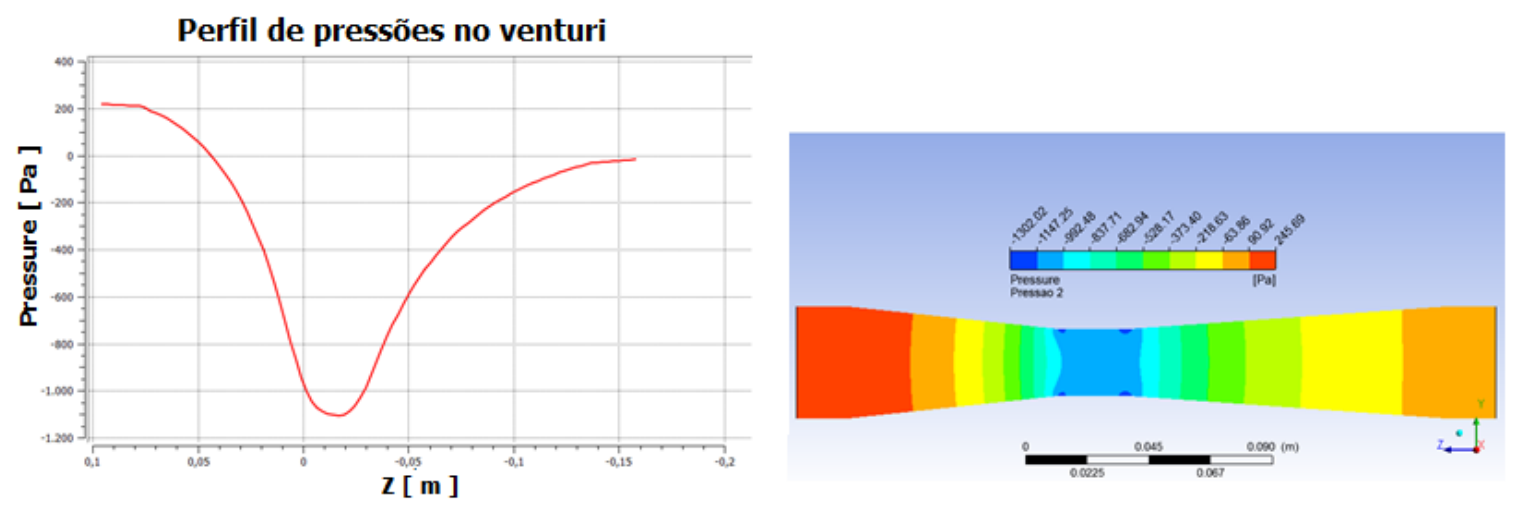

\section{CONCLUSÕES}

A simulação do Venturi do laboratório de Engenharia Química da UFPR por meio de um software de CFD apresentou resultados bastante satisfatórios, com erros menores de $2 \%$, sendo esses resultados condizentes com os já reportados na literatura. Portanto, será possível utilizar as simulações como uma ferramenta didática durante as aulas práticas do curso, já que é possível observar com facilidade as conversões de energia cinética e de pressão. Além disso, as simulações poderão auxiliar o processo de calibração do instrumento diminuindo os gastos e tempo com experimentos.

A malha não estruturada representou melhor a geometria, com erros menores de $1,3 \%$. No entanto, os tempos de simulação para este tipo de malha foram maiores, tonando evidente a vantagem de se utilizar uma malha estruturada, já que os tempos e recursos computacionais exigidos são menores.

Para a malha não estruturada, os erros aumentaram à medida que se aumentou a vazão. Variou-se a intensidade de turbulência, mas os erros continuaram elevados indicando que essa discrepância é devido aos maiores desvios padrão nos últimos pontos experimentais.

\section{REFERÊNCIAS BIBLIOGRÁFICAS}

BIRD, R. B.; STEWART, W. E.; LIGHTFOOT, E. N. Fenômenos de transporte. Rio de Janeiro: Editora LTC, 2004

DIAS, A.; SILVA F. das G. B. da; TIAGO FILHO, G. L. Estudo da Distribuição da Velocidade em Tubo Venturi Utilizando medidas Experimentais e Técnicas de CFD. Revista Brasileira de Recursos Hídricos., v.14, p. 81-92, 2009.

FOX, R. W. Introdução a mecânica dos fluidos. Rio de Janeiro: Editora LTC, 2014

PERRY, R. H.; BENSKOW, L. R.; BEIMESCH, W. E., et al. Perry's Chemical Engineers' Handbook. Nova Iorque: Editora McGraw-Hill, 2008. 